\title{
A short note on approximation properties of Stancu generalization of $q$-Durrmeyer operators
}

\author{
Vishnu Narayan Mishra* and Prashantkumar Patel
}

\section{"Correspondence:}

vishnunarayanmishra@gmail.com

Department of Applied

Mathematics and Humanities,

Sardar Vallabhbhai National

Institute of Technology,

Ichchhanath Mahadev Road, Surat,

Gujarat 395 007, India

\begin{abstract}
In the present paper, we introduce a simple Stancu generalization of $q$-analogue of well-known Durrmeyer operators. We first estimate moments of q-Durrmeyer-Stancu operators. We also establish the rate of convergence as well as Voronovskaja type asymptotic formula for q-Durrmeyer-Stancu operators.
\end{abstract}

\section{Introduction}

In the last decade, the applications of $q$-calculus in the approximation theory is one of the main areas of research. To approximate Lebesgue integrable functions on the interval $[0,1]$, Durrmeyer introduced the integral modification of the well-known Bernstein polynomials. In 1981, Derriennic [1] first studied these operators in detail. After the $q$-analogue of Bernstein polynomials by Phillips [2], Gupta and Heping [3] introduced $q$-Durrmeyer operators. Several other researchers have studied in this direction and obtained different approximation properties of many operators $[4,5]$. In the present article, we propose the $q$-analogue of the Stancu generalization of Durrmeyer operators and study the convergence behavior. We have used notations of $q$-calculus as given in [6-8].

We set $p_{n k}(q ; x)=\left(\begin{array}{l}n \\ k\end{array}\right)_{q} x^{k}(1-x)_{q}^{n-k}, p_{\infty k}(q ; x)=\frac{x^{k}}{(1-q)^{k}[k] q !}(1-x)_{q}^{\infty}$.

Phillips [2] proposed the following $q$-Bernstein polynomials, which for each positive integer $n$ and $f \in C[0,1]$ are defined as

$$
B_{n, q}(f ; x)=\sum_{k=0}^{n} f\left(\frac{[k]_{q}}{[n]_{q}}\right) p_{n k}(q ; x)
$$

In 2005, Derriennic [9] introduced a $q$-analogue of the Durrmeyer operators and has established some approximation properties of the $q$-Durrmeyer operators. After couple of years, Gupta [10] studied $q$-analogue of Durrmeyer operators and discussed approximation properties of the following $q$-Durrmeyer operators: For $f \in C[0,1]$,

$$
\begin{aligned}
D_{n, q}(f ; x) & =[n+1]_{q} \sum_{k=0}^{n} q^{-k} p_{n k}(q ; x) \int_{0}^{1} f(t) p_{n k}(q ; q t) d_{q} t \\
& =\sum_{k=0}^{n} A_{n k}(f) p_{n k}(q ; x) ; \quad 0 \leq x \leq 1 .
\end{aligned}
$$

๑ 2013 Mishra and Patel; licensee Springer. This is an Open Access article distributed under the terms of the Creative Commons Attribution License (http://creativecommons.org/licenses/by/2.0), which permits unrestricted use, distribution, and reproduction in any medium, provided the original work is properly cited. 
Recently, Ibrahim et al. [11, 12] introduced Stancu generalization of certain operators and discussed its approximation properties. Motivated by such type operators, we introduce the Stancu type generalization of the $q$-Durrmeyer operators (1) for $0 \leq \alpha \leq \beta$, which is defined as follows:

$$
\begin{aligned}
D_{n, q}^{\alpha, \beta}(f ; x) & =[n+1]_{q} \sum_{k=0}^{n} q^{-k} p_{n k}(q ; x) \int_{0}^{1} f\left(\frac{[n]_{q} t+\alpha}{[n]_{q}+\beta}\right) p_{n k}(q ; q t) d_{q} t \\
& =\sum_{k=0}^{n} A_{n k}^{\alpha, \beta}(f) p_{n k}(q ; x) ; \quad 0 \leq x \leq 1 .
\end{aligned}
$$

It can be easily verified that in case $q=1, \alpha=0$ and $\beta=0$, the operators defined in (2) reduce to the well-known Durrmeyer operators as defined in [1]. Throughout the present manuscript, the expression $g_{n}(x) \Uparrow g(x)$ means uniform convergence of a sequence $\left\{g_{n}(x)\right\}$ to $g(x)$.

The present note deals with the study of $q$-Durrmeyer-Stancu operators $\left\{D_{n, q}^{\alpha, \beta}(f)\right\}$ for $0<$ $q<1$. First, we estimate the moments for $q$-Durrmeyer-Stancu operators. We also study the rate of convergence as well as asymptotic formula for these operators $\left\{D_{n, q}^{\alpha, \beta}(f)\right\}$. We establish a direct results in terms of $\omega(f, \cdot)$.

\section{Estimation of moments}

In this section, we shall obtain $D_{n, q}^{\alpha, \beta}\left(t^{i} ; x\right), i=0,1, \ldots$

Note that for $s=0,1, \ldots$ and by the definition of $q$-Beta function [7], we have

$$
\begin{aligned}
& \int_{0}^{1} t^{s} p_{n k}(q ; q t) d_{q} t=\frac{q^{k}[n]_{q} ![k+s]_{q} !}{[n+s+1]_{q} ![k]_{q} !} \text { and } \\
& \int_{0}^{1} t^{s} p_{\infty k}(q ; q t) d_{q} t=(1-q)^{s+1} \frac{q^{k}[k+s]_{q} !}{[k]_{q} !} .
\end{aligned}
$$

Lemma 1 We have

$$
\begin{aligned}
D_{n, q}^{\alpha, \beta}(1 ; x)= & 1, \\
D_{n, q}^{\alpha, \beta}(t ; x)= & \frac{[n]_{q}+\alpha[n+2]_{q}+q x[n]_{q}^{2}}{[n+2]_{q}\left([n]_{q}+\beta\right)}, \\
D_{n, q}^{\alpha, \beta}\left(t^{2} ; x\right)= & \frac{q^{3}[n]_{q}^{3}\left([n]_{q}-1\right) x^{2}+\left(\left(q(1+q)^{2}+2 \alpha q^{4}\right)[n]_{q}^{3}+2 \alpha q[3]_{q}[n]_{q}^{2}\right) x}{\left([n]_{q}+\beta\right)^{2}[n+2]_{q}[n+3]_{q}} \\
& +\frac{\alpha^{2}}{\left([n]_{q}+\beta\right)^{2}}+\frac{\left(1+q+2 \alpha q^{3}\right)[n]_{q}^{2}+2 \alpha[3]_{q}[n]_{q}}{\left([n]_{q}+\beta\right)^{2}[n+2]_{q}[n+3]_{q}} .
\end{aligned}
$$

Lemma 2 We have

$$
\begin{aligned}
\delta_{n}(x) & =D_{n, q}^{\alpha, \beta}(t-x, x)=\left(\frac{q[n]_{q}^{2}}{[n+2]_{q}\left([n]_{q}+\beta\right)}-1\right) x+\frac{[n]_{q}+\alpha[n+2]_{q}}{[n+2]_{q}\left([n]_{q}+\beta\right)}, \\
\gamma_{n}(x) & =D_{n, q}^{\alpha, \beta}\left((t-x)^{2}, x\right) \\
& =\frac{q^{4}[n]_{q}^{4}-q^{3}[n]_{q}^{3}-2 q[n]_{q}^{2}[n+3]_{q}\left([n]_{q}+\beta\right)+[n+2]_{q}[n+3]_{q}\left([n]_{q}+\beta\right)^{2}}{\left([n]_{q}+\beta\right)^{2}[n+2]_{q}[n+3]_{q}} x^{2}
\end{aligned}
$$




$$
\begin{aligned}
& +\frac{q(1+q)^{2}[n]_{q}^{3}+2 q \alpha[n]_{q}^{2}[n+3]_{q}-\left(2[n]_{q}+2 \alpha[n+2]_{q}\right)[n+3]_{q}\left([n]_{q}+\beta\right)}{\left([n]_{q}+\beta\right)^{2}[n+2]_{q}[n+3]_{q}} x \\
& +\frac{(1+q)[n]_{q}^{2}+2 \alpha[n]_{q}[n+3]_{q}}{\left([n]_{q}+\beta\right)^{2}[n+2]_{q}[n+3]_{q}}
\end{aligned}
$$

Remark 1 [10] By simple computation, it can easily be verified that

$$
D_{n, q}\left(t^{m} ; x\right)=\frac{[n+1]_{q} !}{[n+m+1]_{q} !} \sum_{k=0}^{n}[k+1]_{q}[k+2]_{q} \cdots[k+m]_{q} p_{n k}(q ; x), \quad r \geq 1 .
$$

Using $[k+s]_{q}=[s]_{q}+q^{s}[k]_{q}$, we get $[k+1]_{q}[k+2]_{q} \cdots[k+m]_{q}=\prod_{s=1}^{m}\left([s]_{q}+q^{s}[k]_{q}\right)=$ $\sum_{s=1}^{m} c_{s}(m)[k]_{q}^{s}$, where $c_{s}(m)>0, s=0,1,2, \ldots, m$ are constants independent of $k$. Hence, $D_{n, q}\left(t^{m} ; x\right)=\frac{[n+1]_{q} !}{[n+m+1]_{q} !} \sum_{s=1}^{m} c_{s}(m) \sum_{k=0}^{n}[k]_{q}^{s} p_{n k}(q ; x)=\frac{[n+1]_{q} !}{[n+m+1]_{q} !} \sum_{s=1}^{m} c_{s}(m)[n]_{q}^{s} B_{n, q}\left(t^{s} ; x\right)$.

Remark 2 For all $m \in \mathbf{N} \cup\{0\}, 0 \leq \alpha \leq \beta$, we have the following recursive relation for the images of the monomials $t^{m}$ under $D_{n, q}^{\alpha, \beta}\left(t^{m} ; x\right)$ in terms of $D_{n, q}\left(t^{j} ; x\right) ; j=0,1,2, \ldots, m$, as

$$
\begin{aligned}
D_{n, q}^{\alpha, \beta}\left(t^{m} ; x\right) & =\sum_{j=0}^{m}\left(\begin{array}{c}
m \\
j
\end{array}\right) \frac{[n]_{q}^{j} \alpha^{m-j}}{\left([n]_{q}+\beta\right)^{m}} D_{n, q}\left(t^{j}, x\right) \\
& =\sum_{j=0}^{m}\left(\begin{array}{c}
m \\
j
\end{array}\right) \frac{[n]_{q}^{j} \alpha^{m-j}}{\left([n]_{q}+\beta\right)^{m}} \frac{[n+1]_{q} !}{[n+j+1]_{q} !} \sum_{s=1}^{j} c_{s}(m)[n]_{q}^{s} B_{n, q}\left(t^{s} ; x\right) .
\end{aligned}
$$

Since $c_{s}(m)>0$ for $s=1,2, \ldots, m$ and $B_{n, q}\left(t^{s} ; x\right)$ is a polynomial of degree $\leq \min (s, n)$ (see [13]), we get $D_{n, q}^{\alpha, \beta}\left(t^{m} ; x\right)$ is a polynomial of degree $\leq \min (m, n)$.

\section{Convergence of $q$-Durrmeyer-Stancu operators}

Theorem 1 Let $q_{n} \in(0,1]$. Then the sequence $\left\{D_{n, q_{n}}^{\alpha, \beta}(f)\right\}$ convergence to $f$ uniformly on $[0,1]$ for each $f \in C[0,1]$ if and only if $\lim _{n \rightarrow \infty} q_{n}=1$.

The proof of the above theorem follows along the lines of [10, Theorem 2], thus we omit the details.

Let $q \in(0,1)$ be fixed. We define $D_{\infty, q}^{\alpha, \beta}(f, 1)=f(1)$ and for $x \in(0,1)$

$$
\begin{aligned}
D_{\infty, q}^{\alpha, \beta}(f ; x) & =\frac{1}{1-q} \sum_{k=0}^{\infty} p_{\infty k}(q ; x) q^{-k} \int_{0}^{1} f\left(\frac{[n]_{q} t+\alpha}{[n]_{q}+\beta}\right) p_{\infty k}(q ; q t) d_{q} t \\
& =\sum_{k=0}^{\infty} A_{\infty k}^{\alpha, \beta}(f) p_{\infty k}(q ; x) .
\end{aligned}
$$

Using the fact that [14], we have

$$
\begin{aligned}
& \sum_{k=0}^{\infty} p_{\infty k}(q ; x)=1, \quad \sum_{k=0}^{\infty}\left(1-q^{k}\right) p_{\infty k}(q ; x)=x, \quad \text { and } \\
& \sum_{k=0}^{\infty}\left(1-q^{k}\right)^{2} p_{\infty k}(q ; x)=x^{2}+(1-q) x(1-x) .
\end{aligned}
$$


Using (3) and (4), it is easy to prove that

$$
\begin{aligned}
D_{\infty, q}^{\alpha, \beta}(1 ; x)=1, & D_{\infty, q}^{\alpha, \beta}(t ; x)=\frac{[n]_{q}(1+q(x-1))+\alpha}{[n]_{q}+\beta} \\
D_{\infty, q}^{\alpha, \beta}\left(t^{2} ; x\right)= & \frac{[n]_{q}^{2} q^{4} x^{2}+\left([n]_{q}^{2}\left(q(1+q)\left(1-q^{2}\right)\right)+2 q \alpha[n]_{q}\right) x}{\left([n]_{q}+\beta\right)^{2}} \\
& +\frac{[n]_{q}^{2}(1-q)^{2}(1+q)+2 \alpha(1-q)[n]_{q}+\alpha^{2}}{\left([n]_{q}+\beta\right)^{2}} .
\end{aligned}
$$

For $f \in C[0,1], t>0$, we define the modulus of continuity $\omega(f, t)$ as follows: $\omega(f, t)=$ $\sup \{|f(x)-f(y)|:|x-y| \leq t, x, y \in[0,1]\}$. We shall show the following theorem.

Theorem 2 Let $0<q<1$ then for each $f \in C[0,1]$ the sequence $\left\{D_{n, q}^{\alpha, \beta}(f ; x)\right\}$ converges to $D_{\infty, q}^{\alpha, \beta}(f ; x)$ uniformly on $[0,1]$. Furthermore, $\left\|D_{n, q}^{\alpha, \beta}(f)-D_{\infty, q}^{\alpha, \beta}(f)\right\| \leq C_{q}^{\alpha, \beta} \omega\left(f, q^{n}\right)$.

The proof of the above theorem follows along the lines of [10, Theorem 3], thus we omit the details.

Remark 3 We may observe that, for $f(x)=x^{2}$, we have $\left\|D_{n, q}^{\alpha, \beta}(f)-D_{\infty, q}^{\alpha, \beta}(f)\right\| \asymp q^{n} \asymp$ $\omega_{2}\left(f, \sqrt{q^{n}}\right)$, where $A(n) \asymp B(n)$ means that $A(n) \ll B(n)$ and $A(n) \gg B(n)$, and $A(n) \ll B(n)$ means that there exists a positive constant $C$ independent of $n$ such that $A(n) \leq C B(n)$. Hence, the estimate of Theorem 2 is sharp in the following sense: the sequence $q^{n}$ in Theorem 2 cannot be replaced by any other sequence decreasing to zero more rapidly as $n \rightarrow \infty$.

Lemma 3 [15] Let L be a positive linear operator on $C[0,1]$, which reproduces linear functions. If $L\left(t^{2}, x\right)>x^{2} \forall x \in(0,1)$, then $L(f)=f$ if and only if $f$ is linear.

Remark 4 Since $D_{\infty, q}^{\alpha, \beta}\left(t^{2}, x\right)=\frac{[n]_{q}^{2}\left[(1-q)^{2}(1+q)+q(1+2 q)(1-q) x+q^{3}(1-q) x+q^{4} x^{2}\right]+2 \alpha[n]_{q}(1+q(x-1))+\alpha^{2}}{[n]_{q}+\beta}>x^{2}$ for $0<q<1$ consequence of Lemma 3 we have the following:

Theorem 3 Let $0<q<1$ be fixed and let $f \in C[0,1]$. Then $D_{\infty, q}^{\alpha, \beta}(f ; x)=f(x)$ for all $x \in[0,1]$ if and only iff is linear.

Remark 5 Let $0<q<1$ be fixed and let $f \in C[0,1]$. Then the sequence $\left\{D_{n, q}^{\alpha \beta}(f ; x)\right\}$ does not approximate $f(x)$ unless $f$ is linear. This is completely in contrast to the classical Bernstein polynomials, by which $\left\{D_{n}(f ; x)\right\}$ approximates $f(x)$ for any $f \in C[0,1]$.

Theorem 4 For any $f \in C[0,1],\left\{D_{\infty, q}^{\alpha, \beta}(f)\right\}$ converges to $f$ uniformly on $[0,1]$ as $q \rightarrow 1-$.

Next, we establish a Voronovskaja type asymptotic formula for the operators $D_{n, q_{n}}^{\alpha, \beta}$ :

Theorem 5 Let $f$ be bounded and integrable on the interval $[0,1]$, second derivative of $f$ exists at a fixed point $x \in[0,1]$ and $q=q_{n} \in(0,1)$ such that $q_{n} \rightarrow 1$ as $n \rightarrow \infty$, then

$$
\lim _{n \rightarrow \infty}[n]_{q_{n}}\left(D_{n, q_{n}}^{\alpha, \beta}(f ; x)-f(x)\right)=(\alpha+1-(2+\beta) x) f^{\prime}(x)+x(1+x) f^{\prime \prime}(x) .
$$


The proof of the above lemma follows along the lines of [16, Theorem 3]; thus, we omit the details.

\section{Competing interests}

The authors declare that they have no competing interests.

\section{Authors' contributions}

PP computed the moments of the modified operators, established the rate of convergence and Voronovskaja type asymptotic formula. VM conceived of the study and participated in its design and coordination. VM and PP contributed equally to this work. All the authors read and approved the final manuscript.

\section{Acknowledgements}

Dedicated to Prof. Hari M. Srivastava on the occasion of his 72th Birth Anniversary.

Received: 14 January 2013 Accepted: 19 March 2013 Published: 4 April 2013

\section{References}

1. Derriennic, MM: Sur $\ell$ approximation de fonctions integrables sur $[0,1]$ par des polynomes de Bernstein modifies. J. Approx. Theory 32, 325-343 (1981)

2. Phillips, GM: Bernstein polynomials based on q-integers. In: The Heritage of P.L. Chebyshev: a Festschrift in Honour of the 70th Birthday of T.J. Rivlin. Ann. Numer. Math. 4, 511-518 (1997)

3. Gupta, GM, Heping, GM: The rate of convergence of $q$-Durrmeyer operators for $0<q<1$. Math. Methods Appl. Sci. 31(16), 1946-1955 (2008)

4. Khan, $\mathrm{HH}$ : Approximation of Classes of function. PhD thesis, AMU, Aligarh (1974)

5. Acar, T, Gupta, V, Aral, A: Rate of convergence for generalized Szász operators. Bull. Math. Sci. 1, 99-113 (2011)

6. Gasper, G, Rahman, M: Basic Hypergeometric Series. Encyclopedia Math. Appl.. Cambridge University Press, Cambridge (1990)

7. Kac, VG, Cheung, P: Quantum Calculus. Springer, New York (2002)

8. Thomae, J: Beitrage zur Theorie der durch die Heinsche Reihe. J. Reine Angew. Math. 70, 258-281 (1869)

9. Derriennic, MM: Modified Bernstein polynomials and Jacobi polynomials in q-calculus. Rend. Circ. Mat. Palermo 76, 269-290 (2005)

10. Gupta, V: Some approximation properties of q-Durrmeyer operators. Appl. Math. Comput. 197, 172-178 (2008)

11. Ibrahim, B: Approximation by Stancu-Chlodowsky polynomials. Comput. Math. Appl. 59, 274-282 (2010)

12. Ibrahim, B, Atakut, C: On Stancu type generalization of $q$ Baskakov operators. Math. Comput. Model. 52, 752-759 (2010)

13. Phillips, GM: Interpolation and Approximation by Polynomials. CMS Books in Math. Springer, New York (2003)

14. Il'inskii, A, Ostrovska, S: Convergence of generalized Bernstein polynomials. J. Approx. Theory 116(1), 100-112 (2002)

15. Wang, $\mathrm{H}$ : Properties of convergence for $\omega, q$-Bernstein polynomials. J. Math. Anal. Appl. 2, 1096-1108 (2008)

16. Gupta, V, Kim, T: On the rate of approximation by q modified Beta operators. J. Math. Anal. Appl. 377, 471-480 (2011)

doi:10.1186/1687-1812-2013-84

Cite this article as: Mishra and Patel: A short note on approximation properties of Stancu generalization of q-Durrmeyer operators. Fixed Point Theory and Applications 2013 2013:84.

\section{Submit your manuscript to a SpringerOpen ${ }^{\ominus}$ journal and benefit from:}

- Convenient online submission

Rigorous peer review

- Immediate publication on acceptance

- Open access: articles freely available online

- High visibility within the field

- Retaining the copyright to your article 